\title{
Analytical Quality by Design: A Mini Review
}

\author{
AV Ganorkar and KR Gupta* \\ Department of Pharmaceutical Chemistry, Smt. Kishoritai Bhoyar College of Pharmacy, India
}

Received: October 25, 2017; Published: November 01, 2017

*Corresponding author: K.R Gupta, Department of Pharmaceutical Chemistry, Smt. Kishoritai Bhoyar College of Pharmacy, Kamptee, India, Email: krg1903@gmail.com

Abbreviations: QbD: Quality By Design; ATP: Analytical Target Profile; HPLC: High Performance Liquid Chromatography; UV: Ultraviolent Rays; GC: Gas Chromatography; QCA: Critical Quality Attributes; MODR: Method Operational Design Region

\section{Introduction}

According to ICH Q8 guidelines, QBD is "A systematic approach to development that begins with predefined objectives and emphasizes product and process understanding and process control, based on sound science and quality risk management."

\section{What is quality by design?}

QbD incorporates planning, developing formulations and manufacturing procedures which ensures predefined product specifications. In 2002, the FDA announced a new initiative (cGMP for the 21st Century: A Risk based Approach). This initiative intended to modernize the FDAs regulation of pharmaceutical quality, and establish a new regulatory framework focused on $\mathrm{QbD}$ risk management, and quality system. The initiative has made the industry to look beyond quality by testing (QbT) for ensuring product quality and performance. An important aspect of QbD is to know how process and formulation parameters could affect the product characteristics and subsequent optimization of these parameters should be known in order to monitor these parameters online in the production process.

The use of QbD concepts results in a well-understood product and process that consistently delivers its intended performance. The knowledge acquired during development may support the formation of a design space and determines suitable process controls. These same QbD principles have been applied to the development of analytical methods, and are termed "Analytical QbD" (AQbD). Analogous to process QbD, the result of AQbD is a well understood, fit for purpose, and robust method that consistently delivers the intended performance throughout its lifecycle. The broad knowledge obtained from this process is used to establish a method operable design region (MODR), a multidimensional space based on the method factors and settings that provide suitable method performance.

$\mathrm{AQbD}$ helps in development of a robust and cost effective analytical method and facilitate the regulatory flexibility in analytical method i.e. the choice to change method parameters within a method's design space, referred to as the method operable design region (MODR).

\section{Regulatory perspective of AQbD}

Analytical methods are key part of the control strategy in pharmaceutical quality system (ICH Q10). It includes various parameters and attributes related to drug substance and drug product including instrument operating conditions and their associated methods. Though cGMP regulation is in practice from a long time but many pharmaceutical giants are facing quality control related issues that are associated with the risk management system in analytical methods. Therefore looking the dependency of pharmaceutical development and manufacture on robust analytical data, need has come for implementation of AQbD in analytical method development, which is an indicator of quality process, product and robustness throughout the life cycle of the product. Below Figure 1 shows various stages in Qbd/AQbD

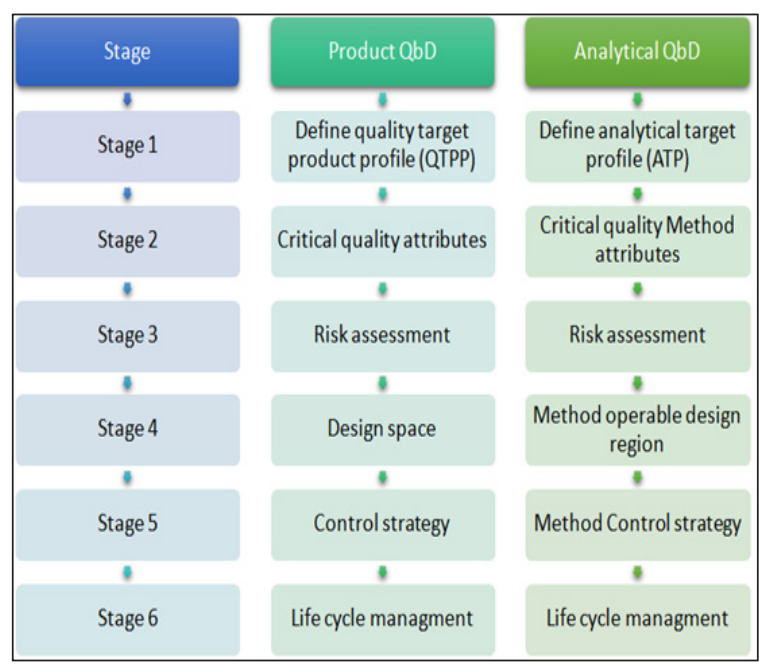

Figure 1: Regulatory perspective of QbDvsAQbD. 


\section{Comparison of Traditional approach VsQbD and AQbD}

Traditional validation methods are usually one time evaluation. As a result chances of method failure during transfer are always high. Also the performance variables are not fully explored and understood. Therefore the below figure 2 summarizes the comparison of traditional and AQbD approach, which aims to handle the shortcomings of the traditional approach based on scientific understanding and knowledge repository.

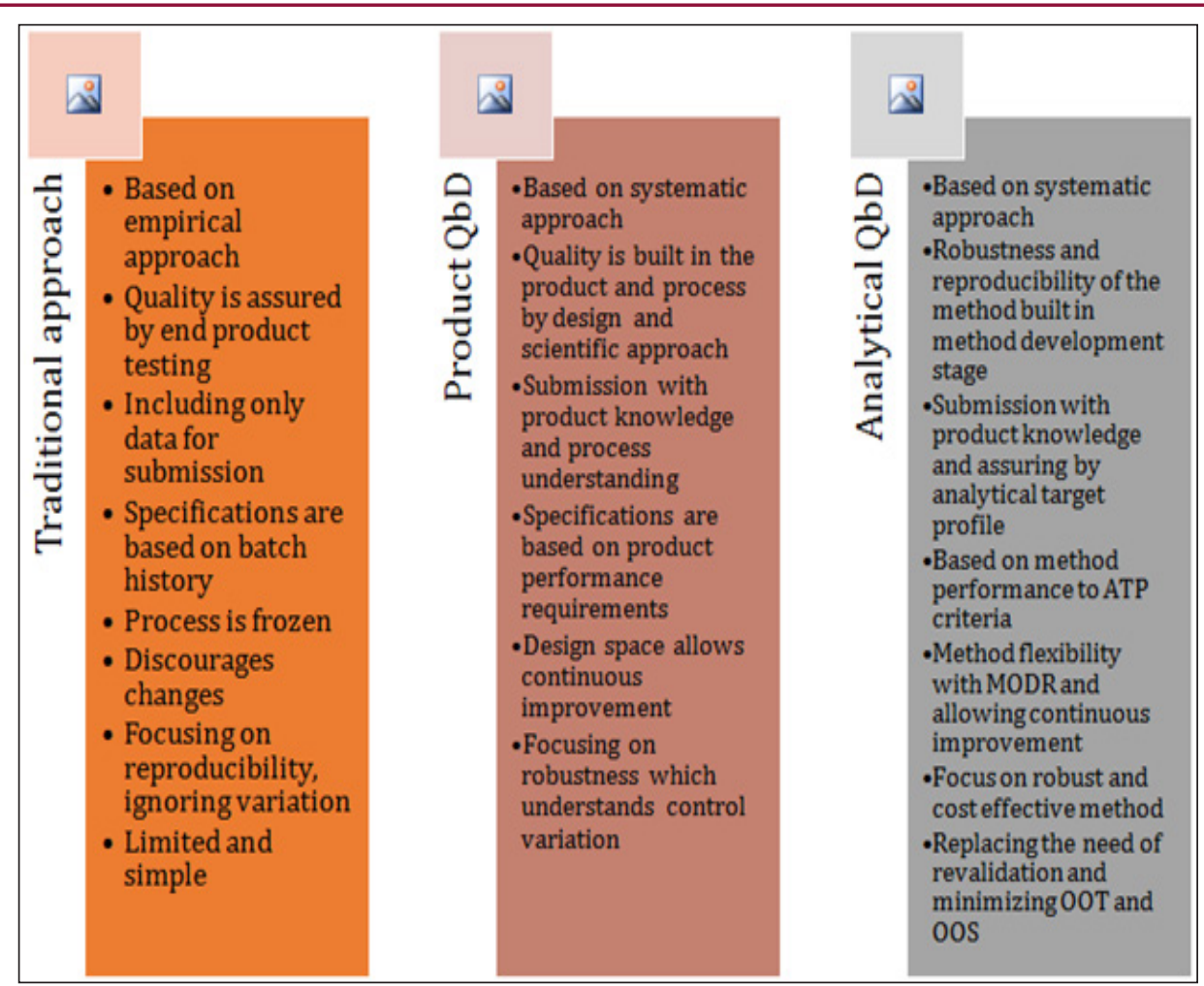

Figure 2: Comparison of Traditional Approach vsQbD and AQbD.

Following are the advantages offered by $\mathrm{AQbD}$ in product development:

1. Scientific understanding of pharmaceutical process and method.

2. It involves product design and process development.

3. Science based risk assessment is carried.

4. Critical quality attributes are identified and their effect on final quality of product is analyzed.

5. It offers robust method or process.

6. It provides required design space for development.

7. Control strategy can be maintained throughout the analysis.

8. It allows continuous improvement till finished steps of method.

9. Flexibility in analysis of API, impurities in dosage forms, stability samples, and metabolites in biological samples.

10. Reduction in variability in analytical attributes for improving the method robustness.

11. Eliminate batch failures.

12. Minimize deviations and costly investigations.
13. Avoid regulatory compliance problems.

14. Better development decisions.

15. Empowerment of technical staff.

16. Smooth process of method transfer to the production level.

\section{Elements of AQbD}

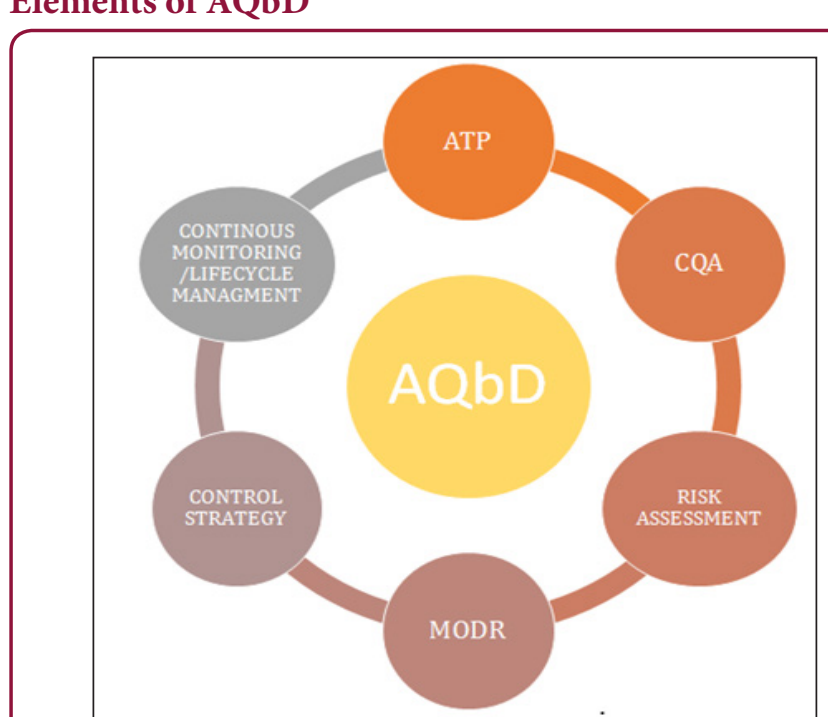

Figure 3: Elements of Analytical Qbd. 
AQbD/qbd comprises of all elements of pharmaceutical development described in ICH Q8 depicted in Figure 3.

\section{Analytical target profile}

ATP is way for method development or it is simply a tool for method development and has been mentioned in the ICH Q8 R (2) guidelines. It defines the method requirements which are expected to be measured that direct the method development process i.e.it is combination of all performance criteria required for the proposed analytical application. An ATP would be developed for each of the traits defined in the control strategy. The ATP defines what the method has to measure (i.e., acceptance criteria) and to what level the measurement is required (i.e., performance level characteristics, such as precision, accuracy, range, sensitivity, and the associated performance criterion). The ATP is defined with the help of knowledge and scientific understanding of the analytical process. Preliminary risk assessment should be carried out for expectation of the method requirements and analytical criticalities. ATP for analytical procedures comprises of

a) Selection of target analytes (API and impurities),

b) Selection of analytical technique (HPTLC, GC, HPLC, Ion Chromatography, chiral HPLC, etc.),

c) Choice of method requirements.

Accuracy and precision are the most important among the performance characteristics that provides the critical information needed to quantify an unknown amount of the substance using the proposed method. A method cannot be accurate and precise without acceptable specificity, linearity over a stated range, sufficient peak resolution for accurate integration, repeatability of injections, etc. To achieve an accurate and precise method the above important characteristics must be evaluated during method development as they provide an extensive data set for setting method controls.

\section{CQA (Critical Quality Attributes)}

ICH Q8 (8) defines CQA as a physical, chemical, biological, or microbiological property or characteristic that should be within an appropriate limit, range, or distribution to ensure the desired product quality. CQA for analytical methods comprises of method attributes and method parameters. CQA can differ from one analytical technique to another.

a) CQA for HPLC (UV or RID) are buffers used in mobile phase, $\mathrm{pH}$ of mobile phase, diluent, column selection, organic modifier and elution method.

b) CQA for GC method is oven temperature and its program, injection temperature, flow rate of gas, sample diluent and concentration.

c) CQA for HPTLC is TLC plate, mobile phase, injection concentration and volume, time taken for plate development, reagent for color development, and detection methods.

Physical and chemical properties of the drug substance and impurities can also describe CQA for analytical method development such as polarity, charged functional groups, solubility, pH value, boiling point and solution stability.

\section{Risk Assessment}

Risk assessment strategy as specified in the ICHQ9 guideline: "it is systematic process for the assessment, control, communication and review of risks to the quality across the product lifecycle". This step is vital in order to reach a confidence level that the method is reliable. Once the technique is identified, AQbD emphases on detailed risk assessment of the factors that may lead to possible variability in the method, like analyst methods, instrument configuration, measurement and method parameters, sample characteristics, sample preparation, and environmental conditions. Traditional method development relied on testing the method after transfer whereas Analytical QbD demands the risk assessment step before method transfer and throughout the product life cycle. According to ICH Q9, risk assessment can be carried out in three steps viz., risk identification, risk analysis and risk evaluation. One of the common ways to perform risk assessment is to use a Fishbone Diagram, also known as Ishikawa. Accordingly the risk factors are classified into the following categories:

a) High Risk Factors: e.g. Sample preparation methodology. These are to be fixed during the Method Development process.

b) Noise Factors: These are subjected to an MSA study. It can be done through staggered cross nested study design and variability plots, ANOVA etc. These factors are subjected to robustness testing.

c) Experimental Factors: e.g. Instrumentation and operation methods. Subjected to ruggedness testing and acceptable range is identified. The third step is Risk Evaluation which is done through Failure mode and effects analysis (FMEA) and the Matrix designs.

\section{MODR (Method Operational Design Region)}

MODR used to develop operational region for routine operation (e.g., analysis time, procedure and limits). In accordance with the requirement of ICH Q8 guidelines, regarding "design space" in product development, method operable design region (MODR) can also be established in method development phase, which could serve as a source for robust and cost effective method. Understanding of method performance regions helps to establish the desired operational conditions. Critical method parameters and analytes sensitivities should be evaluated. MODR is the operating range for the critical method input variable (similar to CQAs) that produces results which consistently meet the goals set out in the ATP. MODR permits the flexibility in various input method parameters to provide the expected method performance criteria and method response without resubmission to FDA. It is based on a science, risk based and multivariate approach to evaluate effects of various factors on method performance.

\section{Method Control Strategy}

Establishing a control strategy is of utmost importance while ensuring that the method is performing as intended on a routine 
basis as goals described in ATP. Basically it's a planned set of controls aimed at minimizing the variability in the process. The strategy is data dependent. Data generated during method development and method verification forms the basis of the control strategy. A factor identified to have risk has to be controlled. More attention is given to the high risk factors. If the risk are low and manageable then the method control strategy can be defined, which generally consists of appropriate system suitability check and verified time to time by having control over it so that method delivers the desirable method attributes. Interestingly, the control strategy of AQbD is not different from the traditional control strategy.

\section{Lifecycle Management}

Going through all the elements of AQbD for a particular analytical method the key steps that ensure fitness of the method for its intended use includes the method validation, verification and Table 1: Few applications applying AQbD approach. transfer. Combining all together is termed as 'lifecycle management of analytical procedure', which commence with establishment of ATP and continues till the methods are in use. The resultant confirmation with respect to ATP is the main focus of performance qualification e.g., precision study at the site of routine use. Continual verification involves activities, which provide the assurance that the method is under control throughout its lifecycle.

\section{Applications OF AQbD}

Numerous applications of AQbD have been reported in the field of Pharmaceutical analysis. We have tried to put some of the examples of chromatographic methods especially liquid chromatography in various areas of analysis that have utilized not all steps shown in Figure 1, but have implemented some aspects of AQbD in their approach. Below Table 1 summarizes some of the application area (Table 1).

\begin{tabular}{|c|c|c|c|c|}
\hline Application Area & Drug & Design Applied For & Author & Journal \\
\hline \multirow[b]{2}{*}{ Impurity Determination } & 1.Vancomycin & Separation of impurity & Alden et. al, & Waters applicaton note \\
\hline & 2.Ziprasidone & $\begin{array}{l}\text { QbD approach to method } \\
\text { development and method } \\
\text { transfer }\end{array}$ & Summer et. al, & Waters applicaton note \\
\hline \multirow[t]{2}{*}{ Column Screening } & & $\begin{array}{l}\text { 1. RP-HPLC Column } \\
\text { Selection }\end{array}$ & Connie et. al, & J. Pharm. Biomed. Anal.2009 \\
\hline & & 2. Column Selection & Kormainy et. al, & J. Pharm. Biomed. Anal.2013 \\
\hline \multirow{2}{*}{ HPLC Method } & $\begin{array}{l}\text { 1.Typical And Atypical } \\
\text { Antipsychotic }\end{array}$ & $\begin{array}{l}\text { Method Selectivity, Anlayte } \\
\text { Retention }\end{array}$ & B. Debrus et. al, & J. Pharm. Biomed. Anal.2013 \\
\hline & 2.Linagliptin & $\begin{array}{l}\text { Method Optimization in } \\
\text { Linagliptin Quantification }\end{array}$ & K. R. Gupta et. al, & EJPMR 2017 \\
\hline \multirow[t]{2}{*}{ Bioanalysis } & 1.Nevirapine & $\begin{array}{l}\text { Nevirapine determination } \\
\text { with liquid-liquid extraction }\end{array}$ & S. Beg et. al, & BioMed. Chromatogr 2015 \\
\hline & 2. Docetaxal & Human plasma & Khurana et. al, & Curr. Pharm. Anal 2015 \\
\hline \multirow{2}{*}{ Dissolution Studies } & 1.Venlafaxine & In-vitro dissolution testing & S. barhate et. al, & Disso. Technol 2016 \\
\hline & 2.Digoxcin & Quantification & Miroslav et. al, & J. Serb. Chem. Soc 2010 \\
\hline \multirow{3}{*}{ Stability Studies } & $\begin{array}{l}\text { 1.Amirodaron HCL And Its } \\
\text { Impurity }\end{array}$ & $\begin{array}{l}\text { Optimized USP Method } \\
\text { Using QbD }\end{array}$ & S. Karmarkar et. al, & $\begin{array}{l}\text { J. Pharm. Biomed. Anal. } \\
2014\end{array}$ \\
\hline & 2.Eberconazole Nitrate & $\begin{array}{l}\text { Method Optimization And } \\
\text { Application To Degradation } \\
\text { Kinetics }\end{array}$ & V.M. Krishna et. al, & J. Saudi. Chem. Soc 2012 \\
\hline & 3.Achyrobichalcon & Method Optimization & J.P. Carini et. al, & Phyto.Chem.Anal.2012 \\
\hline \multirow{2}{*}{ Capillary Electrophoresis } & 1.Levosulpiride & Chiral separation strategy & S. Orlandini et. al, & J. Chromatogr. A 2015 \\
\hline & 2. Amlotriptin & Capillary electrophoresis & S. Orlandini et. al, & J. Chromatogr. A 2014 \\
\hline \multirow{2}{*}{$\begin{array}{l}\text { LC-MS Method } \\
\text { Development }\end{array}$} & 1. Elevitegravir & $\begin{array}{l}\text { Quantification in human } \\
\text { plasma }\end{array}$ & S. Baldelli et. al, & Ther. Drug. Monit 2017 \\
\hline & 2. Fluoxetine & $\begin{array}{l}\text { Quantification in human } \\
\text { plasma }\end{array}$ & M.S. Hasnain et. al, & J. Chromatogr. Sci 2016 \\
\hline
\end{tabular}

\section{Conclusion}

Analytical Quality by Design (AQbD) plays a key role in the pharmaceutical industry for ensuring the method reliability and non-variability in results. The outcome of AQbD is the understanding from method development to method transfer. AQbD tools are ATP, CQA, Method Optimization and Development with DoE, MODR, and Control Strategy with Risk Assessment, Method validation, and continuous improvement. During method development, all potential factors (the inputs) and all critical analytical responses (the outputs) are studied to determine the relationships. Critical analytical factors are identified in an approach that parallels what is described for process development in ICH Q8 and Q9. The AQbD approach plays an active role between analytical scientists during development and operational laboratories as methods are being developed and as factors that lead to potential method failures are identified and controlled. Thus all elements of Aqbd altogether will provide better understanding of the method performance and for its continuous improvement throughout the life cycle. 


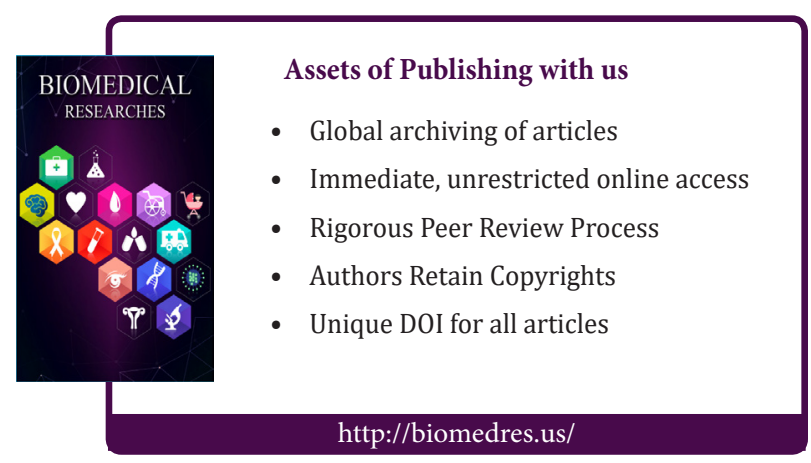

